\title{
Characterization of the DYX2 locus on chromosome 6p22 with reading disability, language impairment, and IQ
}

\author{
John D. Eicher • Natalie R. Powers · Laura L. Miller · Kathryn L. Mueller • Sara Mascheretti • Cecilia Marino • \\ Erik G. Willcutt • John C. DeFries • Richard K. Olson • Shelley D. Smith • Bruce F. Pennington • \\ J. Bruce Tomblin · Susan M. Ring • Jeffrey R. Gruen
}

Received: 12 October 2013 / Accepted: 28 January 2014 / Published online: 9 February 2014

(C) The Author(s) 2014. This article is published with open access at Springerlink.com

\begin{abstract}
Reading disability (RD) and language impairment (LI) are common neurodevelopmental disorders with moderately strong genetic components and lifelong implications. RD and LI are marked by unexpected difficulty acquiring and processing written and verbal language, respectively, despite adequate opportunity and instruction. RD and LI-and their associated deficits-are complex, multifactorial, and often comorbid. Genetic studies have repeatedly implicated the DYX2 locus, specifically the genes DCDC2 and KIAA0319, in RD, with recent studies suggesting they also influence LI, verbal language, and cognition. Here, we characterize the relationship of
\end{abstract}

J. D. Eicher and N. R. Powers contributed to this work equally.

Electronic supplementary material The online version of this article (doi:10.1007/s00439-014-1427-3) contains supplementary material, which is available to authorized users.

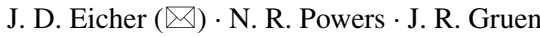

Department of Genetics, Yale University School of Medicine,

New Haven, CT, USA

e-mail: john.eicher@yale.edu

N. R. Powers · J. R. Gruen

Department of Pediatrics, Yale University School of Medicine, New Haven, CT, USA

L. L. Miller · S. M. Ring

MRC Integrative Epidemiology Unit, School of Social

and Community Medicine, University of Bristol, Bristol, UK

\section{K. L. Mueller}

Hearing, Language and Literacy, Murdoch Childrens Institute, Melbourne, Australia

K. L. Mueller · J. B. Tomblin

Department of Communication Sciences and Disorders, The

University of Iowa, Iowa City, IA, USA the DYX2 locus with RD, LI, and IQ. To accomplish this, we developed a marker panel densely covering the $1.4 \mathrm{Mb}$ DYX2 locus and assessed association with reading, language, and IQ measures in subjects from the Avon Longitudinal Study of Parents and Children. We then replicated associations in three independent, disorder-selected cohorts. As expected, there were associations with known RD risk genes KIAA0319 and DCDC2. In addition, we implicated markers in or near other DYX2 genes, including TDP2, ACOT13, C6orf62, FAM65B, and CMAHP. However, the LD structure of the locus suggests that associations within TDP2, ACOT13, and C6orf62 are capturing a previously reported risk variant in KIAA0319. Our results further substantiate the candidacy of KIAA0319 and $D C D C 2$ as major effector genes in DYX2, while proposing FAM65B and CMAHP as new DYX2 candidate genes. Association of DYX2 with multiple neurobehavioral traits

\section{S. Mascheretti · C. Marino}

Child Psychopathology Unit, Scientific Institute, IRCCS Eugenio Medea, Bosisio Parini, Lecco, Italy

\section{Marino}

Centre de Recherche de l'Institut Universitaire en Santé Mentale de Québec, Québec, Canada

C. Marino

Department of Psychiatry and Neuroscience, Université Laval, Québec, Canada

E. G. Willcutt · J. C. DeFries · R. K. Olson

Institute for Behavioral Genetics, University of Colorado, Boulder, CO, USA

E. G. Willcutt · J. C. DeFries · R. K. Olson

Department of Psychology and Neuroscience, University

of Colorado, Boulder, CO, USA 
suggests risk variants have functional consequences affecting multiple neurological processes. Future studies should dissect these functional, possibly interactive relationships of DYX2 candidate genes.

\section{Introduction}

Communication disorders and learning disabilities are common, and can have long-lasting, adverse effects on affected individuals' academic performance, self-esteem, and socioeconomic outcomes. Specifically, reading disability (RD or dyslexia) and language impairment (LI) affect 5-17 and 5-8 \% of schoolchildren, respectively (Newbury et al. 2010; Pennington and Bishop 2009). RD and LI are characterized by unexpected difficulties with reading and verbal language, respectively, despite adequate educational and socioeconomic opportunity and instruction, as well as otherwise normal development (Pennington and Bishop 2009; Newbury et al. 2010). Written and verbal language are closely related and share several neurocognitive processes, including phonological processing and short-term memory (Catts et al. 2005; Gathercole and Baddeley 1990, 1993; Newbury et al. 2010; Pennington 2006; Pennington and Bishop 2009; Wise et al. 2007). In fact, children with LI are more likely to develop RD than their non-impaired peers (Pennington and Bishop 2009).

$\mathrm{RD}$ and LI are complex disorders with substantial genetic and environmental components (Bishop and Hayiou-Thomas 2008; Viding et al. 2004). For instance, the DYX2 locus on chromosome 6p22 has been consistently implicated in RD (Fig. 1) (Cardon et al. 1994; Deffenbacher et al. 2004; Gayán et al. 1999; Kaplan et al. 2002). Two DYX2 genes, DCDC2 and KIAA0319, have been identified as RD risk genes, with considerable genetic and functional molecular evidence supporting the involvement of each (Couto et al. 2010; Cope et al. 2005; Elbert et al. 2011; Francks et al. 2004; Harold et al. 2006; Lind et al. 2010; Luciano et al. 2007; Marino et al. 2012; Meng et al. 2005; Newbury et al. 2011; Paracchini et al. 2006, 2008;

\section{S. D. Smith}

Departments of Pediatrics and Developmental Neuroscience,

University of Nebraska Medical Center, Omaha, NE, USA

B. F. Pennington

Department of Psychology, University of Denver, Denver, CO, USA

\section{J. R. Gruen}

Investigative Medicine Program, Yale Child Health Research Center, Yale University School of Medicine, New Haven, CT, USA

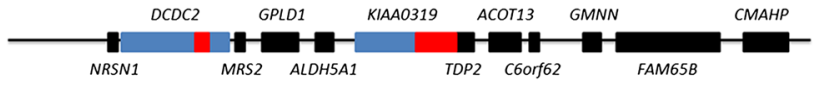

Fig. 1 Schematic of the genes within the DYX2 locus on chromosome 6p22. Genes in blue, DCDC2 and KIAA0319, have replicated associations with written and verbal language phenotypes, namely $\mathrm{RD}$ and LI. Regions in red mark two functional variants, READ1 in $D C D C 2$ and a risk haplotype containing markers in KIAA0319 and $T D P 2$, which have been functionally associated with RD and LI using animal models and molecular techniques

Powers et al. 2013; Scerri et al. 2011; Schumacher et al. 2006; Wilcke et al. 2009; Zhong et al. 2013; Zou et al. 2012). Both $D C D C 2$ and KIAA0319 function in neuronal migration during brain development, as demonstrated by in utero RNAi knockdown studies in rat (Meng et al. 2005; Paracchini et al. 2006). In both genes, putative functional variants appear to be regulatory in nature, as opposed to coding mutations. We previously identified a compound tandem repeat located within the breakpoints of a microdeletion in intron 2 of $D C D C 2$ as a risk variant (Marino et al. 2012; Meng et al. 2005, 2011; Powers et al. 2013). This repeat, known as regulatory element associated with dyslexia 1 (READ1), specifically binds the transcription factor ETV6 and appears capable of modulating expression from the $D C D C 2$ promoter (Meng et al. 2011; Powers et al. 2013). Within KIAA0319, the most strongly associated variant with $\mathrm{RD}$ is a 3-marker risk haplotype that spans approximately the $5^{\prime}$ half of the gene and some of its upstream sequence and neighboring gene TDP2 (Cope et al. 2005; Francks et al. 2004; Paracchini et al. 2006). Both the risk haplotype itself and a putative functional SNP (rs9461045) in linkage disequilibrium with it have been shown to correlate with lower expression of the KIAA0319 gene (Dennis et al. 2009; Elbert et al. 2011). Interestingly, there is evidence that READ1 and the KIAA0319 risk haplotype interact in a non-additive fashion, suggesting that transcriptional co-regulation and interaction may play a key role in the relationship between the DYX2 locus and written/verbal language (Ludwig et al. 2008; Powers et al. 2013).

Although the literature suggests that variants in $D C D C 2$ and KIAA0319 are predominantly responsible for the linkage and association signals from DYX2, other genes and elements may also contribute. The KIAA0319 risk haplotype spans part of the neighboring gene TDP2 (previously known as TTRAP), raising the question of whether the risk haplotype also tags risk variants in TDP2 (Cope et al. 2005; Francks et al. 2004; Paracchini et al. 2006). ACOT13 (previously known as THEM2), a gene adjacent to TDP2, was associated with asymmetry in functional activation of the superior temporal sulcus during reading tasks (Pinel et al. 2012). Genetic linkage analysis showed a linkage peak for full-scale and verbal IQ within ALDH5Al, 
located between KIAA0319 and DCDC2 (Plomin et al. 2004). Verbal IQ is correlated with reading and language skills, so this linkage result may reflect verbal skills measured during IQ testing. ALDH5AI encodes succinic semialdehyde dehydrogenase (SSADH), which influences the neuromodulator $\gamma$-hydroxybutyrate and the metabolism of $\gamma$-amino-butyric acid (GABA). Recent investigations have associated other neurotransmitter factors, including DRD2, DRD4, and COMT, with verbal language and LI, suggesting that neural signaling influences linguistic and cognitive traits (Beaver et al. 2010; Eicher et al. 2013a, b; Landi et al. 2013; Wong et al. 2013). Limited studies of other genes in the DYX2 locus suggest that they may also contribute to reading and language processes and their disorders.

The degree of relatedness of RD and LI indicates that they may share genetic and environmental risk factors. There are reports suggesting that some genes previously associated with RD also associate with LI, and vice versa. Within the DYX2 locus, KIAA0319 has been associated with verbal language as well as reading (Newbury et al. 2011). The most widely studied gene in relation to language, FOXP2, was first implicated in verbal language disorders including dyspraxia of speech and LI, but various studies have expanded these associations with RD, as well as to endophenotypes identified using brain imaging methods (Fisher et al. 1998,; Kaminen et al. 2003; Lai et al. 2001; Peter et al. 2011; Pinel et al. 2012; Wilcke et al. 2011). Similarly, CMIP was first implicated in LI and later associated with reading-related traits (Newbury et al. 2009, 2011; Scerri et al. 2011). Recently, we performed a genome-wide association study (GWAS) on individuals with comorbid RD and LI, which identified ZNF385D as a contributor to processes underlying both disorders (Eicher et al. 2013b). There is strong evidence that RD and LI share genetic components; however, the specific genes and variants contributing to this shared genetic etiology remain largely unknown, as studies have typically been limited in overall number, number of genes examined, and statistical power. In particular, studies of the DYX2 locus have generally not covered the entire DYX2 locus or have tagged it incompletely, and have likely been underpowered to detect single-variant effects.

Therefore, the overall goal of this study was to characterize the associations of the DYX2 locus with RD, LI, and cognition as measured by IQ scores. To accomplish this task, we developed a single-nucleotide polymorphism (SNP) marker panel designed to capture the known common variation of the entire DYX2 locus. We genotyped this DYX2 marker panel in the Avon Longitudinal Study of Parents and Children (ALSPAC) and performed association analysis using reading, language, and cognitive measures collected between the ages of 7 and 9 years. We then replicated our associations in three cohorts selected for either RD or LI. We hypothesized that we would confirm the associations of DCDC2 and KIAAO319 with RD and LI and refine the locations of and possibly identify the variants responsible for these associations. Our results implicating a six-marker haplotype block in linkage disequilibrium with the READ1 element in $D C D C 2$ with Severe RD, and its non-additive genetic interaction with KIAA0319 on reading, verbal language, and IQ performance are presented elsewhere (Powers et al. 2013). Here, we report the additional results of the DYX2 association scan of RD, LI, and IQ.

\section{Methods}

\section{Subjects}

Our discovery cohort in this investigation was the Avon Longitudinal Study of Parents and Children (ALSPAC). The ALSPAC is a population-based birth cohort based in Avon, United Kingdom. Subjects were recruited before birth-a total of 15,458 fetuses were recruited, of whom 14,701 were alive at 1 year of age. Recruitment, participants, and study methodologies are described in detail elsewhere (http://www.bristol.ac.uk/alspac) (Boyd et al. 2012; Golding et al. 2001). The study website contains details of all the data that are available through a fully searchable data dictionary (http://www.bris.ac.uk/alspac/researchers/dataaccess/data-dictionary). DNA samples for genetic analysis were available for 10,259 subjects. Reading, language, and IQ were assessed at ages 7, 8, and 9 years using standardized measures. We excluded subjects with IQ $\leq 75$ on the Wechsler Intelligence Scale for Children (WISC-III) full scale IQ to prevent confounding effects of intellectual disability (Eicher et al. 2013a, b; Powers et al. 2013; Wechsler et al. 1992). To prevent population stratification in genetic analyses, we excluded subjects of non-European descent. Samples with overall genotype call rates $<0.85$ were also excluded from analyses. This resulted in a final sample of 5,579 individuals for language-related analyses and 5,525 individuals for reading-related analyses. Ethical approval was obtained from the ALSPAC Ethics and Law Committee, Local UK Research Ethics Committees, and the Yale Human Investigation Committee.

Following discovery analyses in the ALSPAC, we replicated associated markers in three cohorts specifically recruited for either RD or LI (Table 1). The Iowa LI cohort is composed of $219 \mathrm{LI}$ cases and 209 sex- and age-matched, unrelated controls collected at the University of Iowa. Subjects within the Iowa LI cohort completed a battery of language measures, which were used to derive a composite 
Table 1 Replication cohorts

\begin{tabular}{llll}
\hline & Iowa LI & Colorado RD & Italy RD \\
\hline Cohort-type & Case-control & Family-based & Family-based \\
Number of subjects & 428 & 1,188 & 878 \\
Number of families & N/A & 292 & 304 \\
Disorder & LI & RD & RD \\
Analysis & SVS & TDT (PLINK) & TDT (PLINK) \\
Association conditioned on: & Case-control status & Case-control status, & Case-control status \\
& & discriminant score & Text-, single-word, or \\
Case status determined by: & Composite score on & Composite discriminant & non-word reading \\
& language measures & score on reading tasks & \\
& & &
\end{tabular}

Table 2 ALSPAC phenotype measures

\begin{tabular}{lc}
\hline Measure & Domain \\
\hline Phoneme deletion (PD) age 7 years & Reading \\
Single-word reading (SWR7) age 7 years & Reading \\
Single non-word reading (SNR) age 7 years & Reading \\
Single-word reading (SWR9) age 9 years & Reading \\
Wechsler objective language dimensions (WOLD) verbal comprehension age 8 years & Language \\
Nonword repetition task (NWR) age 8 years & Language \\
Wechsler Intelligence Scale for Children (WISC) full scale IQ (FSIQ) age 8 Years & IQ \\
Wechsler Intelligence Scale for Children (WISC) verbal IQ (VIQ) age 8 Years & IQ \\
Wechsler Intelligence Scale for Children (WISC) performance IQ (PIQ) age 8 Years & IQ \\
\hline
\end{tabular}

language score. This composite score was then dichotomized into case-control status at -1.14 standard deviations (Eicher et al. 2013a; Tomblin et al. 1996; Weismer et al. 2000). The Colorado Learning Disabilities Research Center (CLDRC) or Colorado RD cohort consists of 1,188 individuals within 292 families of twin pairs and their siblings. Families were recruited to the study if at least one member of each twin pair had a history of reading problems (Meng et al. 2005). Within the Colorado RD cohort, RD cases were defined as individuals with a discriminant score below the mean of an age- and sex-matched sample of twins with no school history for RD. The discriminant score is a weighted composite of the reading recognition, comprehension, and spelling subtests of the Peabody Individual Achievement test (PIAT) (Gayán et al. 1999). In the case of monozygotic twins, only one member of each twin pair was used for this study. The Italian cohort consists of 878 individuals in 304 nuclear families; these families were recruited via a proband with clinically diagnosed RD (Marino et al. 2012). Probands were diagnosed with $\mathrm{RD}$ if they scored two or more standard deviations below expected grade level on speed or accuracy on text, word, or nonword reading, had a full-scale IQ of at least 85, and did not have any sensory or neurological disorder (Marino et al. 2012). Ethical approval for recruitment and study methodologies were obtained from the Yale Human Investigation Committee, Institutional Review Boards at the University of Iowa, the University of Denver, University of Colorado-Boulder, University of Nebraska Medical Center, and the Scientific Review Board and the Ethical Committee of the Eugenio Medea Scientific Institute.

ALSPAC reading, language, and IQ measures

In ALSPAC, reading measures used in this investigation included a phoneme deletion task at age 7 years, singleword reading tasks at ages 7 and 9 years, and a single nonword reading task at age 9 years (Table 2). The phoneme deletion task, also known as the Auditory Analysis test, measures phoneme awareness, a core deficit in RD (Rosner and Simon 1971). For this task, the child listens to a word spoken aloud and is then asked to remove a specific phoneme from that word to make a new word. Single-word reading was assessed at age 7 years using the reading subtest of the Wechsler objective reading dimensions (WORD) (Rust et al. 1993). At age 9 years, single-word reading was again assessed by asking the child to read ten real words and ten non-words aloud (Nunes et al. 2003). To examine severe cases (Severe RD), we defined cases as having a score 2 or more standard deviations below the mean on the phoneme 
Table 3 Phenotype definitions for ALSPAC analyses

\begin{tabular}{ll}
\hline & Phenotype definition \\
\hline $\begin{array}{l}\text { Reading (RD) } \\
\text { Severe RD } \\
\text { Moderate RD }\end{array}$ & $\begin{array}{l}\text { 2 Standard deviations below sample mean on the phoneme deletion task } \\
\text { 1 Standard deviation below sample mean on SWR7, SNR, and SWR tasks } \\
\text { Language (LI) }\end{array}$ \\
$\begin{array}{l}\text { Severe LI } \\
\text { Moderate WOLD }\end{array}$ & $\begin{array}{l}\text { 2 Standard deviations below sample mean on either WOLD and/or NWR tasks } \\
\text { Moderate NWR }\end{array}$ \\
1.5 Standard deviations below sample mean on the WOLD task \\
Tognition (IQ) & Quantitative performance on WISC Total IQ task \\
Verbal IQ & Quantitative performance on WISC Verbal IQ task \\
Performance IQ & Quantitative performance on WISC Performance IQ task \\
\hline
\end{tabular}

deletion task (Table 3). We also defined cases with Moderate $\mathrm{RD}$ as scoring at least 1 standard deviation below the mean on single-word reading at age 7 years, single-word reading at age 9 years, and single non-word reading at age 9 years (Table 3 ). We chose a threshold of 1 standard deviation as we included three different measures to isolate individuals with persistently poor decoding skills. We examined different severity levels because past studies in the DYX2 locus have shown differences in genetic association patterns depending on case severity, particularly with KIAA0319 associating with more moderate RD case definitions and $D C D C 2$ with more severe definitions (Paracchini et al. 2008; Powers et al. 2013; Scerri et al. 2011).

Language measures were collected at age 8 years (Table 2). An adaptation of the nonword repetition task (NWR), in which subjects repeated recordings of nonwords, was used to assess short-term phonological memory and processing abilities (Gathercole and Baddeley 1996). Children also completed the Wechsler objective language dimensions (WOLD) verbal comprehension task at age 8 years (Wechsler 1996), where they answered questions about a paragraph read aloud by an examiner describing a presented picture. We chose these measures because individuals with LI are known to perform consistently poorly on NWR and WOLD tasks (Bishop et al. 1996; Newbury et al. 2009). As with RD, we were interested in the association of the DYX2 locus in relation to severity of LI. Here, we defined severe LI cases by scores of 2 or more standard deviations below the sample mean on either language task (severe LI) (Table 3). In contrast, we defined two classes of moderate cases as scoring at least 1.5 standard deviations below the sample mean on either the NWR or WOLD task each task (moderate NWR and moderate WOLD) (Table 3). Verbal IQ, performance IQ, and full scale IQ were assessed at age 8 years, using the Wechsler Intelligence Scale for Children (WISC-III) (Table 2). IQ measures were examined as quantitative traits (Table 2).
Genotyping and genetic analyses

We developed a SNP marker panel in an attempt to capture the common variation in the DYX2 locus. TagSNPs in the locus were selected using the association study design server of Han et al. (2008). The final DYX2 panel contained 195 SNPs with an estimated average power of 83 and $68 \%$ to capture known common and rare variants, respectively, in the DYX2 locus spanning approximately 1.4 Mb. Markers were genotyped on the Sequenom MassARRAY platform (San Diego, CA) following manufacturer's guidelines at the Yale Center for Genome Analysis (West Haven, CT). Briefly, markers were genotyped in nine multiplex reactions of 30-36 markers each, totaling 300 markers (Supplemental Table 1). A subset of markers was not in the DYX2 locus and was not included in the subsequent characterization of the DYX2 locus. In addition to quality control via call rate and Hardy-Weinberg, the histogram plot for each marker was manually evaluated, and a total of 15 markers showing aberrant patterns were excluded. To control for errors in labeling and manipulation of plates, the samples were also genotyped for four sex-determining SNPs in the genes AMELXY and ZNFXY in the pseudoautosomal regions of the $\mathrm{X}$ and $\mathrm{Y}$ chromosomes. These SNPs correctly determined sex for $99.5 \%$ of samples; the remaining samples were excluded.

Markers that deviated substantially from Hardy-Weinberg equilibrium $(p<0.0001)$, or that had an overall call rate $<85 \%$, were not used for genetic analyses. In the discovery ALSPAC cohort, single marker SNP analyses of case-control status and quantitative traits were completed using SNP and variation suite (SVS) v7.6.4 (Bozeman, MT). Linkage disequilibrium was assessed and haplotype blocks were constructed using the four-gamete rule option in HaploView v4.2. Haplotype-based association tests were performed with haplotypes that had frequencies $\geq 1 \%$ using PLINK v1.07 (Barrett et al. 2005; Purcell et al. 
Table 4 Single marker genetic associations with various RD and LI case-control definitions

* Genetic association survives correction for multiple testing

\begin{tabular}{lllllll}
\hline Phenotype & Marker & Gene & BP location & Model & OR (95 \% CI) & $p$ value \\
\hline Severe RD & rs2294691 & TDP2 & $24,652,843$ & Allelic & $2.0(1.3-2.9)$ & 0.00050 \\
Severe RD & rs2294691 & TDP2 & $24,652,843$ & Additive & $1.9(1.3-2.8)$ & 0.00053 \\
Severe RD & rs2294691 & TDP2 & $24,652,843$ & Dominant & $2.3(1.5-3.7)$ & $0.00018^{*}$ \\
Severe RD & rs10456309 & KIAA0319 & $24,589,562$ & Recessive & $10.5(2.2-49.5)$ & $0.00020^{*}$ \\
Moderate RD & rs1562422 & CMAHP & $25,044,577$ & Dominant & $1.7(1.2-2.2)$ & 0.00081 \\
Severe LI & rs807694 & DCDC2 & $24,303,383$ & Additive & $1.8(1.3-2.5)$ & 0.00057 \\
Severe LI & rs807694 & DCDC2 & $24,303,383$ & Allelic & $1.8(1.3-2.5)$ & 0.00050 \\
Severe LI & rs807694 & DCDC2 & $24,303,383$ & Dominant & $1.9(1.3-2.7)$ & 0.00062 \\
Moderate WOLD & rs3756814 & C6orf62 & $24,705,835$ & Additive & $0.7(0.6-0.9)$ & 0.00039 \\
Moderate WOLD & rs3756814 & C6orf62 & $24,705,835$ & Allelic & $0.7(0.6-0.9)$ & 0.00047 \\
Moderate WOLD & rs3777663 & ACOT13 & $24,700,235$ & Additive & $0.6(0.5-0.8)$ & 0.00039 \\
Moderate WOLD & rs3777663 & ACOT13 & $24,700,235$ & Allelic & $0.7(0.5-0.8)$ & 0.00041 \\
\hline
\end{tabular}

2007). To correct for multiple testing, we used a Bonferroni threshold of 0.000256 (0.05 divided by 195 markers) for discovery association tests in the ALSPAC cohort. However, associations with $p<0.001$ are also reported for the ALSPAC discovery cohort to indicate suggestive results.

We tested SNPs that had single marker or within-haplotype associations with $p<0.001$ in the ALSPAC for replication in the Iowa LI, Italian RD, and Colorado RD cohorts. Iowa LI was analyzed using SVS v7.6.4 (Bozeman, MT), while the family-based Italian RD and Colorado RD cohorts were examined using PLINK v1.07 (Purcell et al. 2007). We moved suggestive ALSPAC results forward to our replication analyses to emphasize replication of associations over statistical corrections for multiple testing. Replications with $p<0.05$ in the Iowa LI, Italian RD, and Colorado RD cohorts are reported.

\section{Results}

We performed association with DYX2 markers in three separate domains: (1) RD, (2) LI, and (3) IQ. For the sake of clarity, we present our association findings domain-bydomain, with an emphasis on replication as opposed to correction for multiple testing.

\section{RD}

We performed associations with RD using two different severity definitions: (1) Severe RD and (2) Moderate RD (Table 3). For Severe RD, we observed single marker associations with KIAA0319 and TDP2 (Table 4). There was an association of a six-marker haplotype within $D C D C 2$ that is linked to the risk element READ1 and Severe RD that is explored fully in Powers et al. (2013). TDP2 marker rs2294691 did not replicate its association in any of the three replication cohorts (Table 6). However, KIAA0319 marker rs 10456309 did replicate in Iowa LI and Colorado RD cohorts (Table 6). With Moderate RD, there was an association between rs 1562422 near the gene FAM65B and the pseudogene $C M A H P$, which was replicated in the Colorado RD cohort (Tables 4, 6).

\section{LI}

Association tests were performed on three LI phenotypes: (1) severe LI, (2) moderate NWR, and (3) moderate WOLD (Table 3). As with severe $\mathrm{RD}$, there were associations between $D C D C 2$ and Severe LI. The DCDC2 haplotype that associated with severe LI is discussed in Powers et al. (2013). A marker within this DCDC2 haplotype, rs807694, showed association with Severe LI and was replicated in the Iowa LI cohort (Tables 4, 6). With a more moderate case definition, we observed associations with ACOT13 and C6orf62 (Table 4), genes neighboring KIAA0319 and TDP2. Both rs3777663 in ACOT13 and rs3756814 in C6orf62 showed associations in the Italian RD and Iowa LI cohorts (Table 6).

\section{IQ}

We also performed association tests between DYX2 markers and verbal IQ, performance IQ, and full scale IQ (Table 2). Verbal IQ associations included single markers and haplotypes covering the $5^{\prime}$ half of KIAA0319, rs9348646 in FAM65B, and a haplotype spanning ACOT13 and C6orf62, with evidence of replication (Tables 5a, b, 6). There was substantial overlap of DYX2 associations with verbal IQ and associations with RD and LI. This finding may be a result of the high correlations among these traits (Table 7). The associations of DYX2 with performance IQ and full scale IQ were weaker; there were no associations with performance IQ and a single, non-replicated association of full scale IQ with rs2328791, which is located in 
Table 5 Single marker (a) and haplotype-based (b) genetic associations with quantitative measure of cognition

(a) Single marker genetic associations with cognition

\begin{tabular}{|c|c|c|c|c|c|c|}
\hline Phenotype & Marker & Gene & BP location & Model & Slope & $p$ value \\
\hline Verbal IQ & rs9295626 & KIAA0319 & $24,587,339$ & Allelic & 1.40 & 0.00041 \\
\hline Verbal IQ & rs9295626 & KIAA0319 & $24,587,339$ & Additive & 1.39 & 0.00043 \\
\hline Verbal IQ & rs7763790 & KIAA0319 & $24,615,063$ & Allelic & -1.40 & 0.00045 \\
\hline Verbal IQ & rs7763790 & KIAA0319 & $24,615,063$ & Additive & -1.38 & 0.00048 \\
\hline Verbal IQ & rs6935076 & KIAA0319 & $24,644,322$ & Allelic & 1.16 & 0.00049 \\
\hline Verbal IQ & rs6935076 & KIAA0319 & $24,644,322$ & Additive & 1.15 & 0.00052 \\
\hline Verbal IQ & rs9348646 & FAM65B & $24,052,526$ & Allelic & -1.14 & 0.00066 \\
\hline Verbal IQ & rs9348646 & FAM65B & $24,052,526$ & Additive & -1.14 & 0.00066 \\
\hline Full scale IQ & rs2328791 & N/A & $23,736,848$ & Allelic & -1.21 & 0.00066 \\
\hline Full scale IQ & rs2328791 & N/A & $23,736,848$ & Additive & -1.18 & 0.00075 \\
\hline Full scale IQ & rs2328791 & N/A & $23,736,848$ & Recessive & -3.36 & 0.00042 \\
\hline
\end{tabular}

(b) Haplotype-based genetic associations with cognition

\begin{tabular}{lllllr}
\hline Markers & Haplotype & Gene & BP location & Slope & $p$ value \\
\hline rs2817201, rs9295626 & AT & KIAA0319 & $24,585,214,24,587,339$ & 1.42 & 0.000378 \\
rs10456309, rs4576240, rs17307478, & GGTCAC & KIAA0319 & $24,589,562,24,596,478,24,605,024$ & -1.40 & 0.000569 \\
rs9356939, rs7763790, rs6456621 & & & $24,613,354,24,615,063,24,618,511$ & \\
rs6456624, rs6935076, rs2038137, & AGATA & KIAA0319 & $24,639,223,24,644,322,24,645,943$, & 1.81 & $0.0000145^{*}$ \\
rs3756821, rs1883593, rs3212236 & & & $24,646,821,24,647,191,24,648,455$ & \\
rs3777663, rs3756814, rs6931809, & TGTGGA & ACOT13/C6orf62 & $24,700,235,24,705,835,24,706,770$, & -1.56 & 0.000742 \\
rs6916186, rs6933328, rs17491647 & & & $24,708,523,24,710,920,24,713,723$ & \\
\hline
\end{tabular}

* Genetic association survives correction for multiple testing

a large intergenic region telomeric to NRSN1 and DCDC2 (Tables 5a, b, 6).

Linkage disequilibrium within DYX2

In our analyses, we observed replicated associations in the following genes: $D C D C 2, K I A A 0319$, TDP2, ACOT13, C6orf62, FAM65B, and the pseudogene CMAHP. However, as these SNPs are in close proximity to each other, we next assessed linkage disequilibrium (LD) among our marker panel to determine whether the associated SNPs were tagging the same variation in the locus. Our previous work showed that $D C D C 2$ associations tagged READ1 alleles (Powers et al. 2013). Within KIAA0319, there appears to be two clear LD blocks separating the gene into a $5^{\prime}$ half and a $3^{\prime}$ half (Fig. 2). The $5^{\prime}$ half of KIAA0319 is in strong LD with TDP2, ACOT13, and C6orf62, indicating that associations within these genes may be capturing that same variation (Fig. 2). Associations in FAM65B and $C M A H P$ appear to be tagging independent associations (Fig. 3). Although rs1562422 is located intergenic to $F A M 65 B$ and $C M A H P$, this marker is in strong LD with other markers within the $C M A H P$ pseudogene. Integration of our association analyses and LD structure indicate four independent association signals centered on (1)
$D C D C 2$, (2) the $5^{\prime}$ half of KIAA0319, (3) FAM65B, and (4) CMAHP.

\section{Discussion}

In this investigation, we characterized the relationship of the DYX2 locus with RD, LI, and IQ (Fig. 4). Our results confirm the associations of RD risk genes KIAA0319 and $D C D C 2$ with LI. Additionally, we identify $F A M 65 B$ and $C M A H P$ as candidate genes for linguistic traits. Markers within the DYX2 locus showed association with multiple aspects of communication, including RD, LI, and verbal IQ. However, there was a marked absence of DYX2 associations with full scale IQ and performance IQ, suggesting that the DYX2 locus influences language-related processes to a greater extent than general cognition.

The genetic association of DYX2 with RD, LI, and verbal IQ is the latest example of various neurocognitive and communication processes sharing genetic associations. Our group and others have shown that these neurobehavioral traits have common genetic contributors, including variants in FOXP2, KIAA0319, CMIP, ZNF385D, CNTNAP2, and DCDC2 (Eicher et al. 2013b; Newbury et al. 2009, 2011, Pennington and Bishop 2009, Peter et al. 2011; Pinel et al. 
Table 6 Replication of genetic associations in the Iowa, Italian, and Colorado cohorts
${ }^{a}$ These markers are part of the six-marker risk haplotype in $D C D C 2$ fully discussed in Powers et al. (2013)

Bold denotes nominally associated markers

\begin{tabular}{|c|c|c|c|c|c|c|c|c|c|}
\hline \multirow[t]{2}{*}{ Marker } & \multirow[t]{2}{*}{ Gene } & \multicolumn{2}{|c|}{$\begin{array}{l}\text { Iowa case } \\
\text { control }\end{array}$} & \multicolumn{2}{|c|}{ Italy case control } & \multicolumn{2}{|c|}{$\begin{array}{l}\text { Colorado case } \\
\text { control }\end{array}$} & \multicolumn{2}{|c|}{$\begin{array}{l}\text { Colorado discrimi- } \\
\text { nant score }\end{array}$} \\
\hline & & OR & $p$ & OR & $p$ & OR & $p$ & Slope & $p$ \\
\hline rs2328791 & N/A & 1.0 & 0.813 & 1.0 & 1.000 & 0.9 & 0.646 & 0.087 & 0.447 \\
\hline rs33914824 ${ }^{\mathrm{a}}$ & $D C D C 2$ & 2.2 & 0.034 & 0.9 & 0.768 & 1.1 & 0.847 & 0.023 & 0.934 \\
\hline rs807694 & $D C D C 2$ & 1.9 & 0.028 & 0.9 & 0.786 & 0.9 & 0.853 & -0.025 & 0.919 \\
\hline rs707864 ${ }^{\mathrm{a}}$ & $D C D C 2$ & 1.6 & 0.017 & 1.0 & 0.840 & 1.2 & 0.446 & -0.246 & 0.101 \\
\hline rs $10456301^{a}$ & $D C D C 2$ & 0.9 & 0.553 & 1.1 & 0.811 & 1.5 & 0.289 & 0.221 & 0.162 \\
\hline rs16889066 ${ }^{\mathrm{a}}$ & $D C D C 2$ & 1.2 & 0.517 & 1.0 & 0.884 & 1.2 & 0.622 & -0.304 & 0.150 \\
\hline rs9379651 ${ }^{a}$ & $D C D C 2$ & 1.1 & 0.602 & 1.3 & 0.225 & 0.6 & 0.059 & 0.205 & 0.141 \\
\hline rs 2817201 & KIAA0319 & 1.1 & 0.733 & 1.2 & 0.129 & 1.0 & 1.000 & 0.034 & 0.787 \\
\hline rs9295626 & KIAA0319 & 1.1 & 0.579 & 0.6 & 0.0055 & 1.0 & 0.823 & -0.158 & 0.169 \\
\hline rs 10456309 & KIAA0319 & 0.5 & 0.073 & 0.7 & 0.189 & 0.4 & 0.206 & 0.628 & 0.0133 \\
\hline rs 4576240 & KIAA0319 & 1.1 & 0.825 & 1.9 & 0.0027 & 1.1 & 0.862 & -0.052 & 0.754 \\
\hline rs 17307478 & KIAA0319 & 1.0 & 0.996 & 1.3 & 0.292 & 0.8 & 0.555 & 0.039 & 0.803 \\
\hline rs9356939 & KIAA0319 & 4.0 & 0.018 & 0.8 & 0.069 & 1.3 & 0.151 & -0.116 & 0.254 \\
\hline rs 7763790 & KIAA0319 & 1.0 & 0.831 & 1.1 & 0.627 & 1.4 & 0.163 & 0.014 & 0.910 \\
\hline rs6456621 & KIAA0319 & 2.2 & 0.019 & 1.6 & 0.405 & 1.8 & 0.366 & -0.458 & 0.104 \\
\hline rs 3756821 & KIAA0319 & 1.2 & 0.278 & 1.0 & 0.842 & 1.2 & 0.327 & -0.033 & 0.734 \\
\hline rs 1883593 & KIAA0319 & 1.3 & 0.169 & 1.6 & 0.0052 & 1.3 & 0.239 & -0.108 & 0.395 \\
\hline rs 3212236 & KIAA0319 & 1.0 & 0.883 & 1.1 & 0.496 & 0.9 & 0.745 & -0.124 & 0.319 \\
\hline rs2294691 & $T D P 2$ & 1.1 & 0.779 & 1.9 & 0.0578 & 1.4 & 0.491 & -0.290 & 0.247 \\
\hline rs 3777663 & ACOT13 & 0.7 & 0.016 & 0.6 & 0.0052 & 1.0 & 0.908 & 0.101 & 0.345 \\
\hline rs3756814 & C6orf62 & 0.7 & 0.005 & 0.7 & 0.023 & 0.9 & 0.600 & -0.003 & 0.980 \\
\hline rs6931809 & C6orf62 & 1.4 & 0.023 & 1.4 & 0.017 & 1.2 & 0.491 & -0.096 & 0.382 \\
\hline rs6916186 & C6orf62 & 0.9 & 0.757 & 1.2 & 0.413 & 1.2 & 0.547 & 0.112 & 0.490 \\
\hline rs6933328 & C6orf62 & 0.9 & 0.612 & 0.9 & 0.613 & 1.0 & 0.827 & 0.215 & 0.0515 \\
\hline rs17491647 & C6orf62 & 0.8 & 0.155 & 0.7 & 0.104 & 1.0 & 0.901 & 0.042 & 0.709 \\
\hline rs9348646 & FAM65B & 0.9 & 0.358 & 1.1 & 0.535 & 1.4 & 0.144 & -0.415 & 0.00051 \\
\hline rs 1562422 & СМАНР & 1.0 & 0.793 & 1.0 & 0.796 & 0.6 & 0.093 & -0.030 & 0.840 \\
\hline
\end{tabular}

Table 7 Phenotype correlations in the ALSPAC cohort

\begin{tabular}{|c|c|c|c|c|c|c|c|c|c|}
\hline & NWR & WOLD & SWR7 & SWR9 & SNR & PD & FSIQ & VIQ & $\overline{\text { PIQ }}$ \\
\hline NWR & 1 & & & & & & & & \\
\hline WOLD & 0.214 & 1 & & & & & & & \\
\hline SWR7 & 0.403 & 0.259 & 1 & & & & & & \\
\hline SWR9 & 0.351 & 0.202 & 0.722 & 1 & & & & & \\
\hline SNR & 0.306 & 0.149 & 0.660 & 0.708 & 1 & & & & \\
\hline PD & 0.362 & 0.165 & 0.688 & 0.550 & 0.538 & 1 & & & \\
\hline FSIQ & 0.324 & 0.386 & 0.500 & 0.387 & 0.343 & 0.406 & 1 & & \\
\hline VIQ & 0.346 & 0.424 & 0.536 & 0.421 & 0.421 & 0.426 & 0.871 & 1 & \\
\hline PIQ & 0.192 & 0.216 & 0.292 & 0.218 & 0.218 & 0.246 & 0.819 & 0.435 & 1 \\
\hline
\end{tabular}

All correlations $p<0.05$

$N W R$ nonword repetition age 8 years, WOLD Wechsler objective language dimensions verbal comprehension age 8 years, SWR7 single word reading age 7 years, $S W R 9$ single word reading age 9 years, SNR single nonword reading age 9 years, $P D$ phoneme deletion age 7 years, FSIQ full scale IQ age 8 years, VIQ verbal IQ age 8 years, $P I Q$ performance IQ age 8 years 


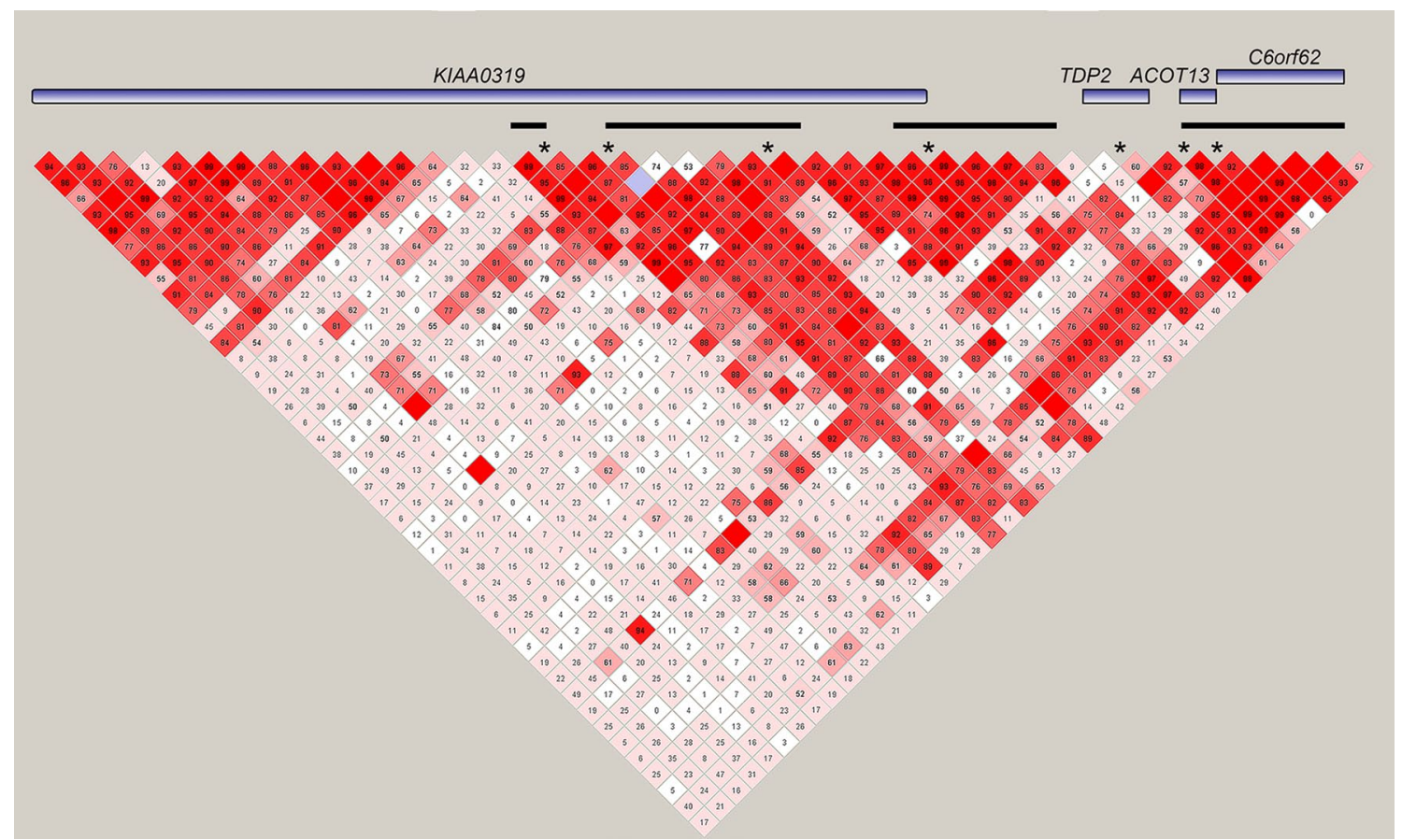

Fig. 2 Linkage disequilibrium between associated markers and haplotypes in KIAA0319, TDP2, ACOT13, and C6Orf62 for RD, LI, and/ or verbal IQ. Asterisks represent single-marker associations, while black bars represent associations with haplotypes. Numbered boxes show LD (as measured by D') between markers. Genes are not drawn to scale; the size of a gene in the diagram depends on the number of markers in our panel that localize to that gene

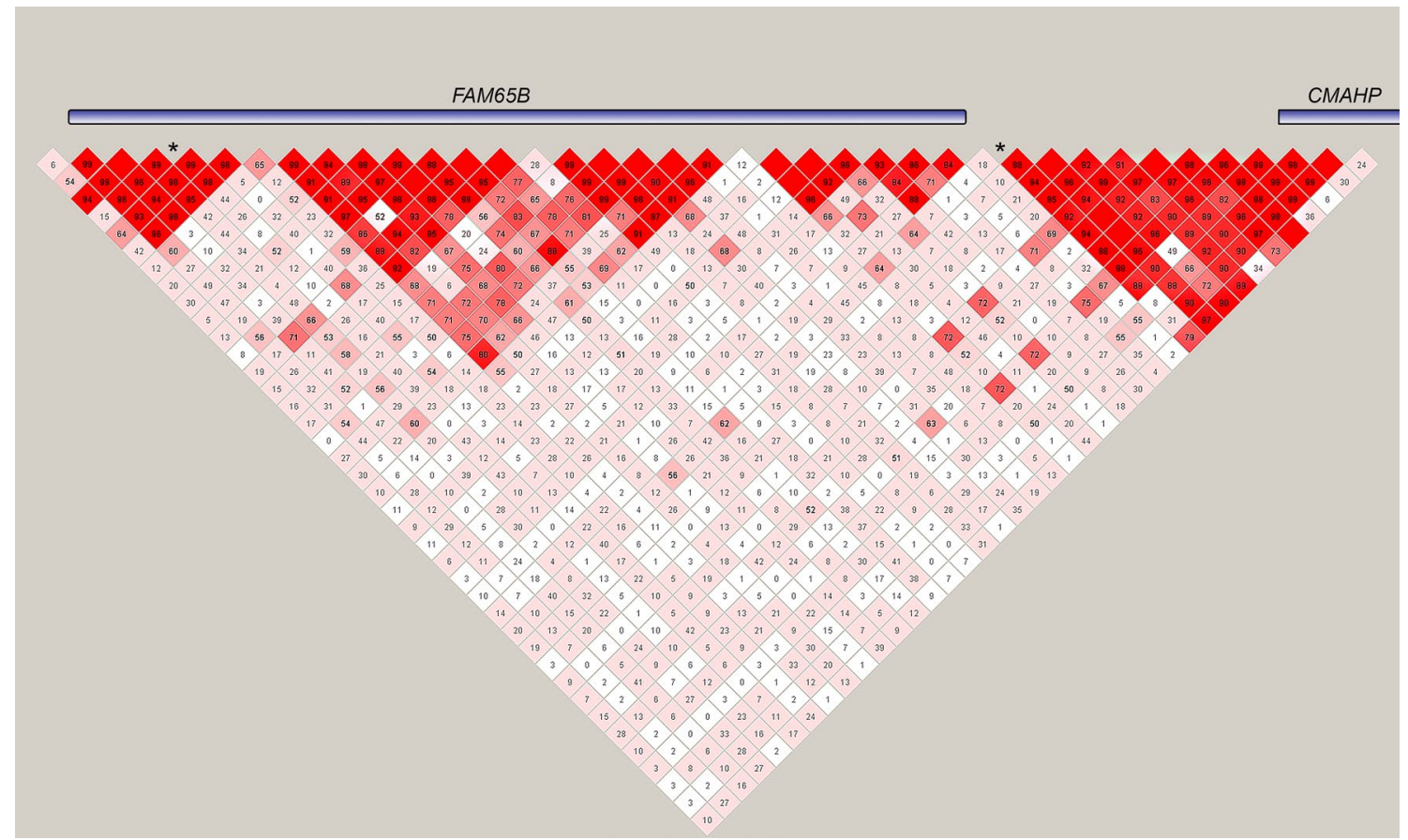

Fig. 3 Linkage disequilibrium between associated markers and haplotypes in $F A M 65 B$ and $C M A H P$ for RD or verbal IQ. Asterisks represent single-marker associations. Numbered boxes show LD (as measured by $\mathrm{D}^{\prime}$ ) between markers. Genes are not drawn to scale; the size of a gene in the diagram depends on the number of markers in our panel that localize to that gene 


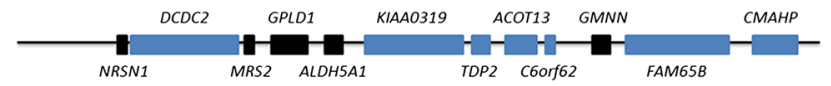

Fig. 4 An updated schematic of genes in our study with markers that show replicated associations with RD, LI, and/or IQ. The list of these genes (shown in blue) has expanded to seven (DCDC2, KIAA0319, TDP2, ACOT13, C6orf62, FAM65B, and CMAHP), although linkage disequilibrium may account for multiple associations (particularly for KIAA0319, TDP2, ACOT13, and C6orf62)

2012; Powers et al. 2013; Scerri et al. 2011; Wilcke et al. 2011). The expansion of DYX2's association from reading to include other language-related processes suggests that the causative variants may affect reading and verbal language in a pleiotropic manner, as opposed to one or the other exclusively. Our findings provide additional evidence for a 'generalist genes hypothesis,' which is also supported by a recent genome-wide complex trait analysis (GTCA) of cognitive and learning abilities (Trzaskowski et al. 2013). The strong correlations and relatedness among these neurocognitive measures (Table 7) suggest that these DYX2 genes affect neurocognitive processes central to language learning, which in turn manifest themselves phenotypically in various ways, including reading, language, and cognition.

That multiple DYX2 genes showed association with the phenotypes in this study is interesting, and at first glance somewhat unexpected. One possibility is that one or two genes are not solely responsible for the consistent implication of this locus in reading, language, and cognitive phenotypes. KIAA0319 and DCDC2 are currently considered as the two major risk genes in the DYX2 locus. Both genes have been implicated in both RD and sub-clinical variation in reading performance, using both classical neurobehavioral measures, and more recently, neuroimaging techniques (Eicher and Gruen 2013; Graham and Fisher 2013). Other genes in DYX2 have been associated with RD, although not nearly as often as DCDC2 and KIAA0319. In this study, with a dense SNP panel, we were able to observe associations with other DYX2 elements, including FAM65B and $C M A H P$. It would appear possible that instead of a single gene-or in this case two genes $(D C D C 2$ and KIAA0319)—multiple elements, possibly regulatory in nature, within DYX2, influence language processes. However, DYX2 has repeatedly shown strong linkage to RD, a pattern generally indicative of one or two variants of large effect, and not of multiple independent variants with more modest effect sizes.

Another possible explanation for the number of DYX2 genes associating in this study is LD within the DYX2 locus. In fact, LD likely explains the cluster of associations around KIAA0319, TDP2, ACOT13, and C6orf62. As shown in Fig. 2, two major LD blocks span KIAA0319-one spans the $3^{\prime}$ half of the gene, while the other spans the $5^{\prime}$ region of KIAA0319 as well as ACOT13, TDP2, and part of C6orf62. Nearly all of the associations in this study localize to this $5^{\prime}$ LD block, which also contains the previously reported KIAA0319 RD risk haplotype (Cope et al. 2005; Francks et al. 2004; Paracchini et al. 2006). Because of this LD structure, it is impossible to determine whether the associations in this region are independent or are capturing the same functional variant. We consider the latter possibility the most likely and believe that the associations in this region are likely tagging the same causative variant captured by the KIAA0319 RD risk haplotype. Functional study of this region - particularly of the less studied genes TDP2, ACOT13, and C6orf62-will almost certainly be necessary to determine whether these associations are independent or not.

By contrast, the markers within or near FAM65B and CMAHP appear to be capturing distinct association signals from two different LD blocks (Fig. 3). The SNP rs9348646, which showed association with verbal IQ, is located within an intron of $F A M 65 B$ in one LD block, while rs 1562422 , which showed association with moderate RD, localized to a separate LD block. While rs 1562422 is an intergenic marker located physically between FAM65B and CMAHP, it shows strong LD with markers in CMAHP (Fig. 3). The LD patterns within the DYX2 locus suggest that associations in KIAA0319, TDP2, ACOT13, and C6orf62 are tagging the same causative variant, while rs9348646 in $F A M 65 B$ and rs 1562422 near $C M A H P$ are independent.

The other DYX2 genes, including FAM65B and CMAHP, have been less studied than the established risk genes DCDC2 and KIAA0319. Little is known about FAM65B in terms of biological function; however, there is evidence that FAM65B may influence migration in T lymphocytes (Rougerie et al. 2013). Animal models of DCDC2 and KIAA0319 have implicated these genes in migratory processes, albeit in a neural context. $C M A H P$, which encodes a key enzyme in the synthesis of the sialic acids Neu5Ac and Neu5Gc in other mammals, was rendered a pseudogene in humans by an inactivating microdeletion and subsequent fixation of the inactive allele in early human populations (Chou et al. 1998). Although ACOT13 appears to be tagging variation within KIAA0319, the preliminary functional studies of ACOT13 are intriguing. ACOT13 was recently associated with lower asymmetric activation of the posterior superior temporal sulcus during reading and phonology tasks (Pinel et al. 2012). The protein product encoded by ACOT13 has been co-localized with beta-tubulin on microtubules; microtubule binding is postulated to be important to RD, as $D C D C 2$ contains two doublecortin domains that are thought to bind microtubules (Cheng et al. 2006).

Genes and regulatory elements within the DYX2 locus may contribute interactively to reading and language 
domains, as seen with the apparent non-additive relationship between putative regulatory variants in $D C D C 2$ and KIAA0319 (Powers et al. 2013, Ludwig et al. 2008). These risk variants have been shown to influence gene expression and to interact with each other to substantially influence performance on reading- and language-related tasks. It is likely that a complex network, where regulatory elements interact and co-regulate other DYX2 genes and elements, contributes to reading, language, and cognitive phenotypes. If so, it is likely that the READ1 element in $D C D C 2$ and the causative variant tagged by the KIAA0319 risk haplotype have the strongest effects on gene expression and the ultimate neurocognitive phenotype. Supporting this idea is the fact that so many of the association hits in the study - both single-marker and haplotype-based, and with all three phenotypes-localize to the same LD block as the KIAA0319 risk haplotype. This result, together with the KIAA0319 risk haplotype's association with reduced KIAA0319 expression and its synergistic interaction with a regulatory element in an intron of $D C D C 2$, strongly suggests the presence of at least one regulatory variant in this region that influences KIAA0319 expression. The locations of the only other independent hits in the locus (aside from READ1 in DCDC2) - an intron of FAM65B and downstream of a pseudogene-may suggest additional regulatory regions that influence gene expression. Thus, any roles FAM65B and CMAHP play in RD and LI may be of small effect and modulatory in nature. Though much further work is needed, we postulate, based on these and previous results, that DCDC2 and KIAA0319 are the major effector genes responsible for DYX2's influence on RD and LI risk and that alteration of gene expression levels or patterns is the mechanism by which this effect is exerted.

In our study design, we emphasized replication of genetic associations in independent cohorts, as opposed to reliance on statistical corrections for multiple testing, for validation of associations in the ALSPAC discovery cohort. The replications of genetic association with our neurocognitive traits of interest, particularly in the varied cohorts in this investigation, provide strong evidence that the results of this study are not due to type I error. However, we also report uncorrected $p$ values and a statistical threshold correcting for 195 genetic markers (threshold of 0.000256) to present the reader with the context of our findings in terms of multiple testing. Nonetheless, our three replication cohorts were not identical and had inherent differences to each other and relative to the discovery cohort that may have prevented replication. These differences included (1) the disorder each cohort was selected for (RD vs. LI vs. unselected), (2) severity of case definition and recruitment, and (3) country of recruitment (UK vs. US vs. Italy), and language spoken (English vs. Italian). Iowa LI and Colorado RD had moderate case definitions, which may be more comparable to Moderate RD, Moderate NWR, and Moderate WOLD case definitions in ALSPAC. On the other hand, Italy RD used a more severe case cutoff of 2 standard deviations, which may be more comparable to severe RD and severe LI case definitions in ALSPAC. Regardless, our observation of multiple replicated associations throughout the DYX2 locus increases confidence in these results.

In summary, our analyses indicate four association signals for RD, LI, and Verbal IQ in the DYX2 locus: $D C D C 2$, KIAA0319, FAM65B, and the pseudogene CMAHP. Our association results within the DCDC2 and KIAA0319 (including TDP2, ACOT13, and C6orf62) areas are in LD with two previously reported risk variants: the READ1 regulatory element in $D C D C 2$ and the KIAA0319 risk haplotype in KIAA0319 and TDP2. These results point strongly, albeit circumstantially, to variation in gene expression as a mediator of DYX2's effect on reading and language phenotypes. As these variants appear to demonstrate pleiotropy, the role of DYX2 in other neurobehavioral disorders, including attention deficit-hyperactivity disorder, autism spectrum disorders, and speech-sound disorder, awaits full characterization to determine the potentially wide range of effects the DYX2 locus has upon the brain and behavior.

Acknowledgments We thank all the families and participants who took part in these studies. We also wish to acknowledge the midwives for their help in recruiting them, and the whole ALSPAC team, which includes interviewers, computer and laboratory technicians, clerical workers, research scientists, volunteers, managers, receptionists and nurses. The UK medical research council and the Wellcome Trust (Grant ref: 092731) and the University of Bristol provide core support for the ALSPAC. This research was specifically funded by the National Institutes of Health (Grant ref: R01 NS043530 [NRP, JRG], P50 HD027802 [EGW, JCD, RKO, SDS, BFP, JRG] and F31 DC012270 [JDE]), the Italian Ministry of Health (SM, CM), the National Health and Medical Research Council (APP1023493) (KLM), the Victorian Government Operational Infrastructure Support Program (KLM), the Manton Family Foundation (JDE, NRP, JRG). The collaboration between JRG and CM rose during a multidisciplinary summer symposium held in Como, Italy, 2004 convened by The Dyslexia Foundation to which we are grateful. We are also grateful to the Yale Center for Genome Analysis, specifically Irina Tikhonova and Anna Rogers, for genotyping services.

Open Access This article is distributed under the terms of the Creative Commons Attribution License which permits any use, distribution, and reproduction in any medium, provided the original author(s) and the source are credited.

\section{References}

Barrett JC, Fry B, Maller J, Daly MJ (2005) Haploview: analysis and visualization of $\mathrm{LD}$ and haplotype maps. Bioinformatics 21:263-265

Beaver KM, Delisi M, Vaughn MG, Wright JP (2010) Association between the A1 allele of the DRD2 gene and reduced verbal abilities in adolescence and early adulthood. J Neural Transm 117(7):827-830 
Bishop DV, Hayiou-Thomas ME (2008) Heritability of specific language impairment depends on diagnostic criteria. Genes Brain Behav 7(3):365-372

Bishop DV, North T, Donlan C (1996) Nonword repetition as a behavioural marker for inherited language impairment: evidence from a twin study. J Child Physiol Psychiatry 37:391-403

Boyd A, Golding J, Macleod J, Lawlor DA, Fraser A, Henderson J, Molloy L, Ness A, Ring S, Davey Smith G (2012) Cohort profile: the 'children of the $90 \mathrm{~s}$ '- the index offspring of the Avon Longitudinal Study of Parents and Children. Int J Epidemiol 42(1):111-127

Cardon LR, Smith SD, Fulker DW, Kimberling WJ, Pennington BF, DeFries JC (1994) Quantitative trait locus for reading disability on chromosome 6. Science 266(5183):276-279

Catts HW, Adlof SM, Hogan TP, Weismer SE (2005) Are specific language impairment and dyslexia distinct disorders? J Speech Lang Hear Res 48(6):1378-1396

Cheng Z, Bao S, Shan X, Xu H, Gong W (2006) Human thioesterase superfamily 2 (hTHEM2) is co-localized with beta-tubulin onto the microtubule. Biochem Biophys Res Commun 350(4):850-853

Chou HH, Takematsu H, Diaz S, Iber J, Knickerson E, Wright KL, Muchmore EA, Nelson DL, Warren ST, Varki A (1998) A mutation in human CMP-sialic acid hydroxylase occurred after the Homo-Pan divergence. Proc Natl Acad Sci USA 95(20):11751-11756

Cope N, Harold D, Hill G, Moskvina V, Stevenson J, Holmans P, Owen MJ, O'Donovan MC, Williams J (2005) Strong evidence that KIAA0319 on chromosome $6 \mathrm{p}$ is a susceptibility gene for developmental dyslexia. Am J Hum Genet 76(4):581-591

Couto JM, Livne-Bar I, Huang K, Xu Z, Cate-Carter T, Feng Y, Wigg K, Humphries T, Tannock R, Kerr EN, Lovett MW, Bremner R, Barr CL (2010) Association of reading disabilities with regions marked by acetylated H3 histones in KIAA0319. Am J Med Genet B Neuropsychatr Genet 153B(2):447-462

Deffenbacher KE, Kenyon JB, Hoover DM, Olson RK, Pennington BF, DeFries JC, Smith SD (2004) Refinement of the 6p21.3 quantitative trait locus influencing dyslexia: linkage and association analyses. Hum Genet 115(2):128-138

Dennis MY, Paracchini S, Scerri TS, Prokunina-Olsson L, Knight JC, Wade-Martins R, Coggill P, Beck S, Green ED, Monaco AP (2009) A common variant associated with dyslexia reduces expression of the KIAA0319 gene. PLoS Genet 5(3):e1000436

Eicher JD, Gruen JR (2013) Imaging-genetics in dyslexia: connecting risk genetic variants to brain neuroimaging and ultimately to reading impairments. Mol Genet Metab. doi:10.1016/j.ymgme.2013.07.001

Eicher JD, Powers NR, Cho K, Miller LL, Mueller KL, Ring SM, Tomblin JB, Gruen JR (2013a) Associations of prenatal nicotine exposure and the dopamine related genes ANKK1 and DRD2 to verbal language. PLoS One 8(5):e63762

Eicher JD, Powers NR, Miller LL, Akshoomoff N, Amaral DG, Bloss CS, Libiger O, Schork NJ, Darst BF, Casey BJ, Chang L, Ernst T, Frazier J, Kaufmann WE, Keating B, Kenet T, Kennedy D, Mostofsky S, Murray SS, Sowell ER, Bartsch H, Kuperman JM, Brown TT, Hagler DJ Jr, Dale AM, Jernigan TL, St. Pourcain B, Davey Smith G, Ring SM, Gruen JR, Pediatric Imaging, Neurocognition, and Genetics Study (2013b) Genome-wide association study of shared components of reading disability and language impairment. Genes Brain Behav 12(8):792-801

Elbert A, Lovett MW, Cate-Carter T, Pitch A, Kerr EN, Barr CL (2011) Genetic variation in the KIAA0319 $5^{\prime}$ region as a possible contributor to dyslexia. Behav Genet 41(1):77-89

Fisher SE, Vargha-Khadem F, Watkins KE, Monaco AP, Pembrey ME (1998) Localisation of a gene implicated in a severe speech and language disorder. Nat Genet 18(2):168-170

Francks C, Paracchini S, Smith SD, Richardson AJ, Scerri TS, Cardon LR, Marlow AJ, MacPhie IL, Walter J, Pennington BF, Fisher SE,
Olson RK, DeFries JC, Stein JF, Monaco AP (2004) A 77-kilobase region of chromosome $6 \mathrm{p} 22.2$ is associated with dyslexia in families from the United Kingdom and from the United States. Am J Hum Genet 75(6):1046-1058

Gathercole S, Baddeley AD (1990) Phonological memory deficits in language disordered children: is there a causal connection? J Mem Lang 29:336-360

Gathercole SE, Baddeley AD (1993) Working memory and language. Lawrence Erlbaum, Mahwah

Gathercole SE, Baddeley AD (1996) The children's test of nonword repetition. The Psychological Corportation, London

Gayán J, Smith SD, Cherny SS, Cardon LR, Fulker DW, Brower AM, Olson RK, Pennington BF, DeFries JC (1999) Quantitative-trait locus for specific language and reading deficits on chromosome 6p. Am J Hum Genet 64(1):157-164

Golding J, Pembrey M, Jones R, ALSPAC Study Team (2001) ALSPAC - the Avon Longitudinal Study of Parents and Children. I. Study methodology. Paediatr Perinat Epidemiol 15(1):74-87

Graham SA, Fisher SE (2013) Decoding the genetics of speech and language. Curr Opin Neurobiol 23(1):43-51

Han B, Kang HM, Seo MS, Zaitlen N, Eskin E (2008) Efficient association study design via power-optimized tag SNP selection. Ann Hum Genet 72(Pt 6):834-847

Harold D, Paracchini S, Scerri T, Dennis M, Cope N, Hill G, Moskvina V, Walter J, Richardson AJ, Owen MJ, Stein JF, Green ED, O’Donovan MC, Williams J, Monaco AP (2006) Further evidence that the KIAA0319 gene confers susceptibility to developmental dyslexia. Mol Psychiatry 11(12):1085-1091

Kaminen N, Hannula-Jouppi K, Kestilä M, Lahermo P, Muller K, Kaaranen M, Myllyluoma B, Voutilainen A, Lyytinen H, NopolaHemmi J, Kere J (2003) A genome scan for developmental dyslexia confirms linkage to chromosome $2 \mathrm{p} 11$ and suggests a new locus on 7q32. J Med Genet 40(5):340-345

Kaplan DE, Gayán J, Ahn J, Won TW, Pauls D, Olson RK, DeFries JC, Wood F, Pennington BF, Page GP, Smith SD, Gruen JR (2002) Evidence for linkage and association with reading disability on 6p21.3-22. Am J Hum Genet 70(5):1287-1298

Lai CS, Fisher SE, Hurst JA, Vargha-Khadem F, Monaco AP (2001) A forkhead-domain gene is mutated in a severe speech and language disorder. Nature 413(6855):519-523

Landi N, Frost SJ, Mencl WE, Preston JL, Jacobsen LK, Lee M, Yrigollen C, Pugh KR, Grigorenko EL (2013) The COMT Val/ Met polymorphism is associated with reading-related skills and consistent patterns of functional neural activation. Dev Sci 16(1):13-23

Lind PA, Luciano M, Wright MJ, Montgomery GW, Martin NG, Bates TC (2010) Dyslexia and DCDC2: normal variation in reading and spelling is associated with DCDC2 polymorphisms in an Australian population sample. Eur J Hum Genet 18(6):668-673

Luciano M, Lind PA, Duffy DL, Castles A, Wright MJ, Montgomery GW, Martin NG, Bates TC (2007) A haplotype spanning KIAA0319 and TTRAP is associated with normal variation in reading and spelling ability. Biol Psychiatry 62:811-817

Ludwig KU, Roeske D, Schumacher J, Schulte-Korne G, Konig IR, Warnke A, Plume E, Ziegler A, Remschmidt H, Muller-Myhsok B, Nothen MM, Hoffman P (2008) Investigation of interaction between DCDC2 and KIAA0319 in a large German dyslexia sample. J Neural Transm 115(11):1587-1589

Marino C, Meng H, Mascheretti S, Rusconi M, Cope N, Giorda R, Molteni M, Gruen JR (2012) DCDC2 genetic variants and susceptibility to developmental dyslexia. Psychiatr Genet 22(1):25-30

Meng H, Smith SD, Hager K, Held M, Liu J, Olson RK, Pennington BF, DeFries JC, Gelernter J, O'Reilly-Pol T, Somlo S, Skudlarski P, Shaywitz SE, Shaywitx BA, Marchione K, Wang Y, Paramasiyam M, LoTurco JJ, Page GP, Gruen JR (2005) DCDC2 is 
associated with reading disability and modulates neuronal development in the brain. Proc Natl Acad Sci USA 102:17053-17058

Meng H, Powers NR, Tang L, Cope NA, Zhang PX, Fuleihan R, Gibson C, Page GP, Gruen JR (2011) A dyslexia-associated variant in DCDC2 changes gene expression. Behav Genet 41(1):58-66

Newbury DF, Winchester L, Addis L, Paracchini S, Buckingham LL, Clark A, Cohen W, Cowie H, Dworzynski K, Everitt A, Goodyer IM, Hennessy E, Kindley AD, Miller LL, Nasir J, O'Hare A, Shaw D, Simkin Z, Simonoff E, Slonims V, Watson J, Ragoussis J, Fisher SE, Seckl JR, Helms PJ, Bolton PF, Pickles A, ContiRamsden G, Baird G, Bishop DV, Monaco AP (2009) CMIP and ATP2C2 modulate phonological short-term memory in language impairment. Am J Hum Genet 85(2):264-272

Newbury DF, Fisher SE, Monaco AP (2010) Recent advances in the genetics of language impairment. Genome Med 2(1):6

Newbury DF, Paracchini S, Scerri TS, Winchester L, Addis L, Richardson AJ, Walter J, Stein JF, Talcott JB, Monaco AP (2011) Investigation of dyslexia and SLI risk variants in reading- and language-impaired subjects. Behav Genet 41(1):90-104

Nunes T, Bryant P, Olssen J (2003) Learning morphological and phonological spelling rules: an intervention study. Sci Stud Read 7(3):289-307

Paracchini S, Thomas A, Castro S, Lai C, Paramasivam M, Wang Y, Keating BJ, Taylor JM, Hacking DF, Scerri T, Francks C, Richardson AJ, Wade-Martins R, Stein JF, Knight JC, Copp AJ, Loturco J, Monaco AP (2006) The chromosome 6p22 haplotype associated with dyslexia reduces the expression of KIAA0319, a novel gene involved in neuronal migration. Hum Mol Genet 15(10): $1659-1666$

Paracchini S, Steer CD, Buckingham LL, Morris AP, Ring S, Scerri T, Stein J, Pembrey ME, Ragoussis J, Golding J, Monaco AP (2008) Association of the KIAA0319 dyslexia susceptibility gene with reading skills in the general population. Am J Psychiatry 165(12):1576-1584

Pennington BF (2006) From single to multiple deficit models of developmental disorders. Cognition 101(2):385-413

Pennington BF, Bishop DV (2009) Relations among speech, language, and reading disorders. Annu Rev Psychol 60:283-306

Peter B, Raskind WH, Matsushita M, Lisowski M, Vu T, Berninger VW, Wijsman EM, Brkanac Z (2011) Replication of CNTNAP2 association with nonword repetition and support for FOXP2 association with timed reading and motor activities in a dyslexia family sample. J Neurodev Disord 3(1):39-49

Pinel P, Fauchereau F, Moreno A, Barbot A, Lathrop M, Zelenika D, Le Bihan D, Poline JB, Bourgeron T, Dehaene S (2012) Genetics variants of FOXP2 and KIAA0319/TTRAP/THEM2 locus are associated with altered brain activation in distinct languagerelated regions. J Neurosci 32(3):817-825

Plomin R, Turic DM, Hill L, Turic DE, Stephens M, Williams J, Owen MJ, O'Donovan MC (2004) A functional polymorphism in the succinate-semialdehyde dehydrogenase (aldehyde dehydrogenase 5 family, member A1) gene is associated with cognitive ability. Mol Psychiatry 9(6):582-586

Powers NR, Eicher JD, Butter F, Kong Y, Miller LL, Ring SM, Mann M, Gruen JR (2013) Alleles of a polymorphic ETV6 binding site in DCDC2 confer risk of reading and language impairment. Am J Hum Genet 93(1):19-28

Purcell S, Neale B, Todd-Brown K, Thomas L, Ferreira MA, Bender D, Maller J, Sklar P, de Bakker PI, Daly MJ, Sham PC (2007) PLINK: a tool set for whole-genome association and population-based linkage analyses. Am J Hum Genet 81(3):559-575

Rosner J, Simon DP (1971) The auditory analysis test: an initial report. J Learn Disabil 4(384):40-48

Rougerie P, Largeteau Q, Megrelis L, Carrette F, Lejeune T, Toffali L, Rossi B, Zeghouf M, Cherfils J, COnstantin G, Laudanna C, Bismuth G, Mangeney M, Delon J (2013) Famb65b is a new transcriptional target of FOXO1 that regulates RhoA signaling for $\mathrm{T}$ lymphocyte migration. J Immunol 190(2):748-755

Rust J, Golombok S, Trickey G (1993) WORD: Wechsler objective reading dimensions manual. Psychological Corporation, Sidcup

Scerri TS, Morris AP, Buckingham LL, Newbury DF, Miller LL, Monaco AP, Bishop DV, Paracchini S (2011) DCDC2, KIAA0319 and CMIP are associated with reading-related traits. Biol Psychiatry 70:237-245

Schumacher J, Anthoni H, Dahdouh F, König IR, Hillmer AM, Kluck N, Manthey M, Plume E, Warnke A, Remschmidt H, Hülsmann J, Cichon S, Lindgren CM, Propping P, Zucchelli M, Ziegler A, Peyrard-Janvid M, Schulte-Körne G, Nöthen MM, Kere J (2006) Strong genetic evidence of DCDC2 as a susceptibility gene for dyslexia. Am J Hum Genet 78(1):52-62

Tomblin JB, Records N, Zhang X (1996) A system for the diagnosis of specific language impairment in kindergarten children. J Speech Hear Res 39(6):1284-1294

Trzaskowski M, Davis OS, DeFries JC, Yang J, Visscher PM, Plomin R (2013) DNA evidence for strong genome-wide pleiotropy of cognitive and learning abilities. Behav Genet 43(4):267-273

Viding E, Spinath FM, Price TS, Bishop DV, Dale PS, Plomin R (2004) Genetic and environmental influence on language impairment in 4-year-old same-sex and opposite-sex twins. J Child Psychol Psychiatry 45(2):315-325

Wechsler D (1996) Wechsler objective language dimensions (WOLD). Psychological Corporation, London

Wechsler D, Golombok S, Rust J (1992) WISC-III UK: Wechsler Intelligence Scale for Children. Psychological Corporation, Sidcup

Weismer SE, Tomblin JB, Zhang X, Buckwalter P, Chynoweth JG, Jones M (2000) Nonword repetition performance in school-age children with and without language impairment. J Speech Lang Hear Res 43(4):865-878

Wilcke A, Weissfuss J, Kirsten H, Wolfram G, Boltze J, Ahnert P (2009) The role of gene DCDC2 in German dyslexics. Ann Dyslexia 59(1):1-11

Wilcke A, Ligges C, Burkhardt J, Alexander M, Wolf C, Quente E, Ahnert P, Hoffmann P, Becker A, Muller-Myhsok B, Cichon S, Boltze J, Kirsten H (2011) Imaging genetics of FOXP2 in dyslexia. Eur J Hum Genet 20(2):224-229

Wise JC, Sevcik RA, Morris RD, Lovett MW, Wolf M (2007) The relationship among reception and expressive vocabulary, listening comprehension, pre-reading skills, word identification skills, and reading comprehension by children with reading disabilities. J Speech Lang Hear Res 50(4):1093-1099

Wong PC, Ettlinger M, Zheng J (2013) Linguistic grammar learning and DRD2-TAQ-1A polymorphism. PLoS One 8(5):e64983

Zhong R, Yang B, Tang H, Zou L, Song R, Zhu LQ, Miao X (2013) Meta-analysis of the association between DCDC2 polymorphisms and risk of dyslexia. Mol Neurobiol 47(1):435-442

Zou L, Chen W, Shao S, Sun Z, Zhong R, Shi J, Miao X, Song R (2012) Genetic variant in KIAA0319, but not in DYX1C1, is associated with risk of dyslexia: an integrated meta-analysis. Am J Med Genet B Neuropsychiatr Genet 159B(8):970-976 